CALT-68-1960

October 1994

\title{
On the Classification of Fusion Rings
}

\author{
Doron Gepner 周 \\ Anton Kapustin ${ }^{\dagger}$ \\ Department of Physics, Mathematics and Astronomy \\ California Institute of Technology \\ Pasadena, CA 91125
}

\begin{abstract}
The fusion rules and modular matrix of a rational conformal field theory obey a list of properties. We use these properties to classify rational conformal field theories with not more than six primary fields and small values of the fusion coefficients. We give a catalogue of fusion rings which can arise for these field theories. It is shown that all such fusion rules can be realized by current algebras. Our results support the conjecture that all rational conformal field theories are related to current algebras.
\end{abstract}

* On leave of absence from Weizmann Institute, Israel

${ }^{\dagger}$ Work supported in part by the U.S.Dept. of Energy under Grant No. DE-FG03-92-ER40701 
Rational conformal field theories have attracted considerable attention starting with the seminal work of BPZ [1]. One main question is the problem of the classification of rational conformal field theories (RCFT). It was suggested [2] that all such theories stem from current algebras via the coset construction or various extended algebras. The first step is to try to establish if all fusion rings of RCFT stem from current algebras. This question was discussed in [3, 4, 5]; in particular in [3] it was shown that all fusion rings with three or less primaries originate from current algebras. In this note we wish to address this question by making a computer classification of fusion rings of RCFT for low number of primary fields and a low value for the fusion coefficients.

Our results can be summarized as follows. We studied RCFT with the number of primary fields $n \leq 6$ and the fusion coefficients $N_{i j}^{k} \leq 100,10,3,1$ for $n=3,4,5,6$ respectively. We find that all the theories found in this range can be obtained from current algebras, and that no "exotic" theories exist.

Our starting point is the fusion rules and the modular transformations of the RCFT, following the connection found in ref. [6], between the two. In an RCFT these are required to obey the following set of properties:

1) The fusion rules form an associative commutative ring. The structure constants are non-negative integers, $N_{i j}^{k}$, which obey $N_{i j}^{k} N_{k l}^{m}=N_{i l}^{k} N_{k j}^{m}$.

2) There is a symmetric bilinear form $b_{i j}$ such that $b_{i j}=0$, except for $i=\bar{j}$, where $\bar{j}$ is some permutation of $j$, and where $b_{i, \bar{i}}=1$. The structure constant $N_{i j k}=b_{i m} N_{j k}^{m}$, is symmetric in all three indices. $N_{i j}^{0}=b_{i j}$, where 0 denotes the identity field.

3) The matrix $S$ of modular transformations is a symmetric unitary matrix whose order is the number of primary fields and is related to the fusion rules by

$$
N_{i j}^{k}=\sum_{m} \frac{S_{i m} S_{j m} S_{k m}^{\dagger}}{S_{0 m}} .
$$

The matrix $S$ obeys $\left(S^{2}\right)_{i j}=b_{i j}$, and implements the modular transformation $\tau \rightarrow \frac{-1}{\tau}$. In addition, the diagonal matrix $T=\exp \left[2 \pi i\left(\Delta_{p}-c / 24\right)\right]$, where $\Delta_{p}$ is the dimension of the primary field $p$ and $c$ is the central charge, implements the modular transformation $\tau \rightarrow \tau+1$ and obeys $(S T)^{3}=b$.

4) The structure constants and the dimensions obey the equations [7]

$$
\left(\alpha_{i} \alpha_{j} \alpha_{k} \alpha_{l}\right)^{N_{i j k l}}=\prod_{r} \alpha_{r}^{N_{i j k l, r}}
$$

where,

$$
\alpha_{r}=\exp \left(2 \pi i \Delta_{r}\right), \quad N_{i j k l}=N_{i j}^{\bar{m}} N_{k l}^{m}, \quad N_{i j k l, r}=N_{i j}^{r} N_{k l r}+N_{j k}^{r} N_{i l r}+N_{i k}^{r} N_{j l r} .
$$

An RCFT obeys additional properties such as the braid group representation [8]. However, on the basis of our study of theories with low number of primary fields we suggest that the 
properties (1-4) fully characterize an RCFT, and that any commutative ring satisfying these properties is the fusion ring of some full fledged RCFT. The question is thus to classify rings obeying the properties (1-4).

This problem is very difficult to settle completely. We thus resort to a computer study which can be readily done for a small number of primary fields and a small value for the fusion coefficients $N_{i j k}$. The following procedure was employed to generate fusion rings. We select one primary field, denoted by ' 1 ', different from the identity. We than fix the structure constants $N_{1 j}^{k}$, subject to the condition that $N_{1 j k}$ is fully symmetric. We can always find such values for the rest of the fusion coefficients that the fusion ring be associative. Now, in the vast majority of cases, $N_{1 j}^{k}$ determines the full fusion ring, by associativity. Degenerate cases, where this is not so, are treated separately. We run the program over all values of $N_{1 i}^{j}$ consistent with the symmetry of $N_{1 i j}$ and for a fixed small number of primary fields and some upper bound for $N_{1 i}^{j} \leq M$, where $M$ is small.

For each selection we proceed to construct the $S$ matrix by solving the system of equations,

$$
\psi_{1} \psi_{j}=N_{1 j}^{k} \psi_{k}
$$

where $\psi_{i}$ are some complex numbers. This is essentially a linear problem, whose eigenvectors span the $n$-dimensional space where $n$ is the number of primary fields, if $N_{1 i}^{j}$ is a multiplication matrix of a fusion ring. The solutions $\psi_{i}$ are related by property (3) to the $S$ matrix by

$$
\psi_{i}^{(j)}=\frac{S_{i j}}{S_{i 0}}
$$

We thus construct the matrix $S$ by taking the matrix $\psi_{i}^{(j)}$, permuting arbitrarily its columns, and normalizing each column to have norm 1, up to an undetermined \pm 1 sign $\left(S_{0 j}\right.$ must be all real). We then check if the matrix $S$ is symmetric (it is automatically unitary). This is a very stringent condition which rules out all but a very small number of rings. For these we solve the equations (2) to find the possible dimensions, and check for each solution if $(S T)^{3}=b$ is satisfied. This concludes the search for rings satisfying (1-4), except for the degenerate cases. For these we generate the remaining fusion coefficients, check for associativity and symmetry and proceed in the same way.

We present our results in tables (1-5). These tables are a complete catalogue of fusion rings with not more than six primary fields (with the above mentioned limitations on the structure constants). All the fusion rings that we find can be realized in terms of current algebras. We find theories of two types: 1) WZW models [9]; 2) Theories obtained by taking the extended algebra of products of current algebras [10. The fusion rings of WZW theories were described in ref. [11]. The fusion rings of type 2 are closely related to the latter and can be described, for our puposes, as the sub-rings of representations invariant 
under the center of the corresponding WZW model. For example, $S U(3)_{4} / Z_{3}$ which has five primary fields has a fusion ring which is the same as $S U(3)_{4} \mathrm{WZW}$ theory, but with only $Z_{3}$ (color) singlet representations retained. It is remarkable that one can always find realizations for fusion rings with six or less primaries in terms of the RCFTs of these two types. Note that other types of RCFT are known, such as orbifolds [12 and coset models [13]. However, these do not seem to be required for the realizations. It is an interesting question whether this situation persists for more that six primaries.

Note that the same fusion rules correspond, in general, to a number of different modular matrices. In the context of WZW theories, this ambiguity was described in ref [10]. The different $S$ matrices are obtained from each other by some permutation of the columns. It is presently unkown whether all such $S$ matrices are actually realized as modular matrices of some RCFT. For this reason, we cannot list realizations for all the modular matrices that we find.

This brings us to the question if all RCFT can be constructed from current algebras by cosets, orbifolds and so forth. In other words, do current algebras completely classify RCFT? We investigate here a slightly weaker version of this question: are all fusion rings realized by current algebras. Our findings indicate that this is indeed the case. This lends considerable support to the conjecture that all RCFT can be realized in terms of current algebras, and that we essentially know already all RCFT. This is a somewhat disappointing finding. We know that the set of non-rational conformal field theories is very rich, including moduli. It is unfortunate that only current algebras, then, have the vast simplification and beauty of rationality.

Our investigation was carried only up to six primary fields. However, a stronger computer than the desk-top we used can be utilized to carry the search to a higher number of primary fields without any change in the algorythm. We believe that investigation up to ten or so primary fields is quite feasible. We strongly suspect, however, that the main result will persist, and that all fusion rings can be realized in terms of current algebras.

Finally, we hope that this work helps to clarify a question that has been in the air for quite some time. Namely, what are the RCFT and do we already know all of them. In view of the many connections of RCFT to integrable models, solvable lattice systems, etc., this question is of particular interest.

Acknowledgements. We would like to thank S. Cherkis for numerous discussions.

\section{References}

[1] A.A. Belavin, A.M. Polyakov and A.B. Zamolodchikov, Nucl. Phys. B241 (1984) 333 
[2] G. Moore and N. Seiberg, Phys. Lett. B220 (1989) 422

[3] M. Caselle and G. Ponzano, Phys. Lett. B242 (1990) 52, Int. J. Mod. Phys. A5 (1990) 4697

[4] M. Caselle, G. Ponzano and F. Ravanini, Int. J. Mod. Phys. B6 (1992) 2075-2090, and references therein.

[5] W. Eholzer, On the classification of modular fusion algebras, Bonn university preprint BONN-TH-94-18

[6] E. Verlinde, Nucl. Phys. B300 (1988) 360

[7] C. Vafa, Phys. Lett. B206 (1988) 421

[8] J. Fröhlich, In Como 1987 proceedings, Differential Geometry Methods in Theoretical Physics, p. 173-186; B. Schroer, ibid. p. 219-259; J. Fröhlich, Cargese Summer Institute 1987, p. 71-100; L.A. Gaumé, ibid. p. 1-11; G. Moore and N. Seiberg, Trieste spring school 1989, p. 1.

[9] V. Knizhnik and A.B. Zamolodchikov, Nucl. Phys. B247 (1984) 83; E. Witten, Comm. Math. Phys. 92 (1984) 455

[10] D. Gepner, Foundations of Rational Quantum Field Theory I, Caltech preprint CALT-68-1825, November 1992

[11] D. Gepner and E. Witten, Nucl. Phys. B278 (1986) 493

[12] L. Dixon, J.A. Harvey, C. Vafa and E. Witten, Nucl. Phys. B261 (1985) 678; Nucl. Phys. B274 (1986) 285

[13] P. Goddard, A. Kent and D. Olive, Phys. Lett. B152 (1985) 88, Comm. Math. Phys. 103 (1986) 105; D. Kastor, E. Martinec and Z. Qiu, Phys. Lett. B200 (1988) 434; J. Bagger, D. Nemeschansky and S. Yankielowicz, Phys. Rev. Lett. 60 (1988) 389; M. Douglas, G/H Conformal Field Theories, Caltech preprint CALT-68-1453, September 1987 


\begin{tabular}{c|c|c|c} 
& Fusion ring & Central charge mod4 & Realization \\
\hline \hline & $\phi^{2}=1$ & 1,3 & $S U(2)_{1}$ \\
\hline & $\phi^{2}=1+\phi$ & $\frac{4 n+2}{5}, n<5, n \neq 2$ & $S U(2)_{3} / Z_{2}$ \\
\hline
\end{tabular}

Table 1: Fusion rings with two primaries.

\begin{tabular}{c|c|c|c} 
& Fusion ring & Central charge mod4 & Realization \\
\hline \hline$\phi_{1}^{2}=1, \quad \begin{array}{c}\phi_{2}^{2}=1+\phi_{1}, \\
\phi_{1} \phi_{2}=\phi_{2}\end{array}$ & $\frac{2 n+1}{2}, n<4$ & $S U(2)_{2}$ \\
\hline $\begin{array}{c}\phi_{2}^{2}=1+\phi_{1}+\phi_{2}, \\
\phi_{1} \phi_{2}=\phi_{1}+\phi_{2}\end{array}$ & $\frac{4 n}{7}, n<7$ & $S U(2)_{5} / Z_{2}$ \\
\hline $\begin{array}{c}\phi_{1}^{2}=1+\phi_{2}, \\
\phi_{1} \phi_{2}=1\end{array}$ & 2 & $S U(3)_{1}$ \\
\hline
\end{tabular}

Table 2: Fusion rings with three primaries and fusion coefficients $N_{i j k} \leq 100$. 


\begin{tabular}{|c|c|c|}
\hline Fusion ring & $\begin{array}{c}\text { Central charge } \\
\text { mod4 }\end{array}$ & Realization \\
\hline $\begin{array}{c}\phi_{1}^{2}=1, \phi_{2}^{2}=1 \\
\phi_{3}^{2}=1, \phi_{1} \phi_{2}=\phi_{3} \\
\phi_{1} \phi_{3}=\phi_{2}, \phi_{2} \phi_{3}=\phi_{1}\end{array}$ & 0,2 & $\begin{array}{l}S U(2)_{1} \times S U(2)_{1} \\
\quad \text { or } S O(8)_{1}\end{array}$ \\
\hline $\begin{array}{c}\phi_{1}^{2}=1, \phi_{2}^{2}=1+\phi_{2} \\
\phi_{3}^{2}=1+\phi_{2} \\
\phi_{1} \phi_{2}=\phi_{3}, \phi_{1} \phi_{3}=\phi_{2} \\
\phi_{2} \phi_{3}=\phi_{1}+\phi_{3}\end{array}$ & $\begin{array}{c}\frac{2 n+1}{5} \\
n<10 \\
n \neq 2,7\end{array}$ & $S U(2)_{3}$ \\
\hline $\begin{array}{c}\phi_{1}^{2}=1+\phi_{1}, \\
\phi_{2}^{2}=1+\phi_{2}, \\
\phi_{3}^{2}=1+\phi_{1}+\phi_{2}+\phi_{3}, \\
\phi_{1} \phi_{2}=\phi_{3} \\
\phi_{1} \phi_{3}=\phi_{2}+\phi_{3}, \\
\phi_{2} \phi_{3}=\phi_{1}+\phi_{3}\end{array}$ & $\begin{aligned} & \frac{2 n}{5} \\
& n<9 \\
& n \neq 3,5,7\end{aligned}$ & $S U(2)_{3} / Z_{2} \times S U(2)_{3} / Z_{2}$ \\
\hline $\begin{array}{c}\phi_{1}^{2}=1+\phi_{2}, \\
\phi_{2}^{2}=1+\phi_{2}+\phi_{3}, \\
\phi_{3}^{2}=1+\phi_{1}+\phi_{2}+\phi_{3}, \\
\phi_{1} \phi_{2}=\phi_{1}+\phi_{3}, \\
\phi_{1} \phi_{3}=\phi_{2}+\phi_{3}, \\
\phi_{2} \phi_{3}=\phi_{1}+\phi_{2}+\phi_{3}\end{array}$ & $\frac{2}{3}, \frac{10}{3}$ & $S U(2)_{7} / Z_{2}$ \\
\hline $\begin{array}{c}\phi_{1}^{2}=1, \phi_{2}^{2}=\phi_{1} \\
\phi_{3}^{2}=\phi_{1}, \phi_{1} \phi_{2}=\phi_{3} \\
\phi_{1} \phi_{3}=\phi_{2}, \phi_{2} \phi_{3}=1\end{array}$ & 1,3 & $S U(4)_{1}$ \\
\hline
\end{tabular}

Table 3: Fusion rings with four primaries and $N_{i j k} \leq 10$. 


\begin{tabular}{|c|c|c|}
\hline Fusion ring & $\begin{array}{c}\text { Central charge } \\
\bmod 4\end{array}$ & Realization \\
\hline $\begin{array}{c}\phi_{1}^{2}=1+\phi_{2}, \\
\phi_{2}^{2}=1+\phi_{2}+\phi_{4}, \\
\phi_{3}^{2}=1+\phi_{2}+\phi_{3}+\phi_{4}, \\
\phi_{4}^{2}=1+\phi_{1}+\phi_{2}+\phi_{3}+\phi_{4}, \\
\phi_{1} \phi_{2}=\phi_{1}+\phi_{3}, \\
\phi_{1} \phi_{3}=\phi_{2}+\phi_{4}, \\
\phi_{1} \phi_{4}=\phi_{3}+\phi_{4}, \\
\phi_{2} \phi_{3}=\phi_{1}+\phi_{3}+\phi_{4}, \\
\phi_{2} \phi_{4}=\phi_{2}+\phi_{3}+\phi_{4}, \\
\phi_{3} \phi_{4}=\phi_{1}+\phi_{2}+\phi_{3}+\phi_{4}\end{array}$ & $\frac{\frac{4(n+1)}{11},}{n<9}$ & $S U(2)_{9} / Z_{2}$ \\
\hline $\begin{array}{c}\phi_{1}^{2}=1+\phi_{2} \\
\phi_{2}^{2}=1+\phi_{2}+\phi_{4} \\
\phi_{3}^{2}=1+\phi_{2}, \phi_{4}^{2}=1 \\
\phi_{1} \phi_{2}=\phi_{1}+\phi_{3} \\
\phi_{1} \phi_{3}=\phi_{2}+\phi_{4} \\
\phi_{1} \phi_{4}=\phi_{3}, \phi_{2} \phi_{3}=\phi_{1}+\phi_{3} \\
\phi_{2} \phi_{4}=\phi_{2}, \phi_{3} \phi_{4}=\phi_{1}\end{array}$ & 2 & $S U(2)_{4}$ \\
\hline $\begin{array}{c}\phi_{1}^{2}=\phi_{3}, \phi_{2}^{2}=\phi_{4} \\
\phi_{3}^{2}=\phi_{2}, \phi_{4}^{2}=\phi_{1} \\
\phi_{1} \phi_{2}=1, \phi_{1} \phi_{3}=\phi_{4} \\
\phi_{1} \phi_{4}=\phi_{2}, \phi_{2} \phi_{3}=\phi_{1} \\
\phi_{2} \phi_{4}=\phi_{3}, \phi_{3} \phi_{4}=1\end{array}$ & 0 & $S U(5)_{1}$ \\
\hline $\begin{array}{c}\phi_{1}^{2}=1+\phi_{1}+\phi_{2}, \\
\phi_{2}^{2}=1+\phi_{1}+2 \phi_{2}+\phi_{3}+\phi_{4}, \\
\phi_{3}^{2}=\phi_{1}+\phi_{4}, \\
\phi_{4}^{2}=\phi_{1}+\phi_{3}, \\
\phi_{1} \phi_{2}=\phi_{1}+\phi_{2}+\phi_{3}+\phi_{4}, \\
\phi_{1} \phi_{3}=\phi_{2}+\phi_{4}, \\
\phi_{1} \phi_{4}=\phi_{2}+\phi_{3}, \\
\phi_{2} \phi_{3}=\phi_{1}+\phi_{2}+\phi_{3}, \\
\phi_{2} \phi_{4}=\phi_{1}+\phi_{2}+\phi_{4}, \\
\phi_{3} \phi_{4}=1+\phi_{2}\end{array}$ & $\begin{array}{c}\frac{4 n+2}{7} \\
n<7 \\
n \neq 3\end{array}$ & $S U(3)_{4} / Z_{3}$ \\
\hline
\end{tabular}

Table 4: Fusion rings with five primaries and $N_{i j k} \leq 3$. 


\begin{tabular}{|c|c|c|}
\hline Fusion ring & $\begin{array}{c}\text { Central charge } \\
\bmod 4\end{array}$ & Realization \\
\hline $\begin{array}{c}\phi_{1}^{2}=1, \phi_{2}^{2}=2, \\
\phi_{3}^{2}=1+\phi_{2}, \phi_{4}^{2}=1, \\
\phi_{5}^{2}=1+\phi_{2}, \phi_{1} \phi_{2}=\phi_{4}, \\
\phi_{1} \phi_{3}=\phi_{5}, \phi_{1} \phi_{4}=\phi_{2}, \\
\phi_{1} \phi_{5}=\phi_{3}, \phi_{2} \phi_{3}=\phi_{3}, \\
\phi_{2} \phi_{4}=\phi_{1}, \phi_{2} \phi_{5}=\phi_{5}, \\
\phi_{3} \phi_{4}=\phi_{5}, \phi_{3} \phi_{5}=\phi_{1}+\phi_{4}, \\
\phi_{4} \phi_{5}=\phi_{3}\end{array}$ & $\begin{array}{l}\frac{2 n+1}{2} \\
n<4\end{array}$ & $S U(2)_{2} \times S U(2)_{1}$ \\
\hline $\begin{array}{c}\phi_{1}^{2}=1, \phi_{2}^{2}=1+\phi_{3} \\
\phi_{3}^{2}=1+\phi_{2}+\phi_{3} \\
\phi_{4}^{2}=1+\phi_{3} \\
\phi_{5}^{2}=1+\phi_{2}+\phi_{3} \\
\phi_{1} \phi_{2}=\phi_{4}, \phi_{1} \phi_{3}=\phi_{5} \\
\phi_{1} \phi_{4}=\phi_{2}, \phi_{1} \phi_{5}=\phi_{3} \\
\phi_{2} \phi_{3}=\phi_{2}+\phi_{3} \\
\phi_{2} \phi_{4}=\phi_{1}+\phi_{5} \\
\phi_{2} \phi_{5}=\phi_{4}+\phi_{5} \\
\phi_{3} \phi_{4}=\phi_{4}+\phi_{5} \\
\phi_{3} \phi_{5}=\phi_{1}+\phi_{4}+\phi_{5} \\
\phi_{4} \phi_{5}=\phi_{2}+\phi_{3} \\
\end{array}$ & $\begin{array}{c}\frac{2 n+1}{7} \\
n<14 \\
n \neq 3,10\end{array}$ & $S U(2)_{5}$ \\
\hline $\begin{array}{c}\phi_{1}^{2}=1, \phi_{2}^{2}=\phi_{3}, \\
\phi_{3}^{2}=\phi_{2}, \phi_{4}^{2}=\phi_{3}, \\
\phi_{5}^{2}=\phi_{2}, \phi_{1} \phi_{2}=\phi_{4}, \\
\phi_{1} \phi_{3}=\phi_{5}, \phi_{1} \phi_{4}=\phi_{2}, \\
\phi_{1} \phi_{5}=\phi_{3}, \phi_{2} \phi_{3}=1, \\
\phi_{2} \phi_{4}=\phi_{5}, \phi_{2} \phi_{5}=\phi_{1}, \\
\phi_{3} \phi_{4}=\phi_{1}, \phi_{3} \phi_{5}=\phi_{4}, \\
\phi_{4} \phi_{5}=1\end{array}$ & 1,3 & $S U(6)_{1}$ \\
\hline
\end{tabular}

Table 5: Fusion rings with six primaries and $N_{i j k} \leq 1$. 


\begin{tabular}{|c|c|c|}
\hline Fusion ring & $\begin{array}{c}\text { Central } \\
\text { charge } \bmod 4\end{array}$ & Realization \\
\hline $\begin{array}{c}\phi_{1}^{2}=1+\phi_{1}, \phi_{2}^{2}=1+\phi_{3}, \\
\phi_{3}^{2}=1+\phi_{2}+\phi_{3}, \\
\phi_{4}^{2}=1+\phi_{1}+\phi_{3}+\phi_{5}, \\
\phi_{5}^{2}=1+\phi_{1}+\phi_{2}+\phi_{3} \\
+\phi_{4}+\phi_{5} \\
\phi_{1} \phi_{2}=\phi_{4}, \phi_{1} \phi_{3}=\phi_{5}, \\
\phi_{1} \phi_{4}=\phi_{2}+\phi_{4} \\
\phi_{1} \phi_{5}=\phi_{3}+\phi_{5} \\
\phi_{2} \phi_{3}=\phi_{2}+\phi_{3} \\
\phi_{2} \phi_{4}=\phi_{1}+\phi_{5} \\
\phi_{2} \phi_{5}=\phi_{4}+\phi_{5} \\
\phi_{3} \phi_{4}=\phi_{4}+\phi_{5} \\
\phi_{3} \phi_{5}=\phi_{1}+\phi_{4}+\phi_{5} \\
\phi_{4} \phi_{5}=\phi_{2}+\phi_{3} \\
+\phi_{4}+\phi_{5}\end{array}$ & $\begin{array}{c}\frac{4 n+2}{35}, \\
n<35, \\
n \neq 2,3,7,10, \\
12,22,24 \\
27,31,32\end{array}$ & $\begin{array}{c}S U(2)_{3} / Z_{2} \\
\times S U(2)_{5} / Z_{2}\end{array}$ \\
\hline $\begin{array}{c}\phi_{1}^{2}=1+\phi_{1}, \phi_{2}^{2}=1 \\
\phi_{3}^{2}=1+\phi_{2}, \phi_{4}^{2}=1+\phi_{1}, \\
\phi_{5}^{2}=1+\phi_{1}+\phi_{2}+\phi_{4} \\
\phi_{1} \phi_{2}=\phi_{4}, \phi_{1} \phi_{3}=\phi_{5} \\
\phi_{1} \phi_{4}=\phi_{2}+\phi_{4} \\
\phi_{1} \phi_{5}=\phi_{3}+\phi_{5} \\
\phi_{2} \phi_{3}=\phi_{3}, \phi_{2} \phi_{4}=\phi_{1} \\
\phi_{2} \phi_{5}=\phi_{5}, \phi_{3} \phi_{4}=\phi_{5} \\
\phi_{3} \phi_{5}=\phi_{1}+\phi_{4} \\
\phi_{4} \phi_{5}=\phi_{3}+\phi_{5}\end{array}$ & $\begin{array}{c}\frac{2 n+1}{10}, \\
n<20 \\
n \neq 2,7,12,17\end{array}$ & $\begin{array}{c}S U(2)_{3} / Z_{2} \\
\times S U(2)_{2}\end{array}$ \\
\hline $\begin{array}{c}\phi_{1}^{2}=1+\phi_{1}, \phi_{2}^{2}=\phi_{3}, \\
\phi_{3}^{2}=\phi_{2}, \phi_{4}^{2}=\phi_{3}+\phi_{5}, \\
\phi_{5}^{2}=\phi_{2}+\phi_{4} \\
\phi_{1} \phi_{2}=\phi_{4}, \phi_{1} \phi_{3}=\phi_{5}, \\
\phi_{1} \phi_{4}=\phi_{2}+\phi_{4} \\
\phi_{1} \phi_{5}=\phi_{3}+\phi_{5} \\
\phi_{2} \phi_{3}=1, \phi_{2} \phi_{4}=\phi_{5}, \\
\phi_{2} \phi_{5}=\phi_{1}, \phi_{3} \phi_{4}=\phi_{1} \\
\phi_{3} \phi_{5}=\phi_{4}, \phi_{4} \phi_{5}=1+\phi_{1}\end{array}$ & $\begin{array}{l}\frac{4 n+4}{5} \\
n<5\end{array}$ & $S U(3)_{2}$ \\
\hline
\end{tabular}




\begin{tabular}{|c|c|c|}
\hline Fusion ring & $\begin{array}{c}\text { Central } \\
\text { charge mod4 }\end{array}$ & Realization \\
\hline $\begin{array}{c}\phi_{1}^{2}=1+\phi_{2}, \\
\phi_{2}^{2}=1+\phi_{2}+\phi_{4}, \\
\phi_{3}^{2}=1+\phi_{2}+\phi_{4}+\phi_{5}, \\
\phi_{4}^{2}=1+\phi_{2}+\phi_{3} \\
+\phi_{4}+\phi_{5}, \\
\phi_{5}^{2}=1+\phi_{1}+\phi_{2}, \\
+\phi_{3}+\phi_{4}+\phi_{5}, \\
\phi_{1} \phi_{2}=\phi_{1}+\phi_{3}, \\
\phi_{1} \phi_{3}=\phi_{2}+\phi_{4}, \\
\phi_{1} \phi_{4}=\phi_{3}+\phi_{5}, \\
\phi_{1} \phi_{5}=\phi_{4}+\phi_{5}, \\
\phi_{2} \phi_{3}=\phi_{1}+\phi_{3}+\phi_{5}, \\
\phi_{2} \phi_{4}=\phi_{2}+\phi_{4}+\phi_{5}, \\
\phi_{2} \phi_{5}=\phi_{3}+\phi_{4}+\phi_{5}, \\
\phi_{3} \phi_{4}=\phi_{1}+\phi_{3}+\phi_{4}+\phi_{5}, \\
\phi_{3} \phi_{5}=\phi_{2}+\phi_{3}+\phi_{4}+\phi_{5}, \\
\phi_{4} \phi_{5}=\phi_{1}+\phi_{2}+\phi_{3}, \\
+\phi_{4}+\phi_{5}\end{array}$ & $\begin{array}{c}\frac{4 n+2}{13} \\
n<13 \\
n \neq 6\end{array}$ & $S U(2)_{11} / Z_{2}$ \\
\hline $\begin{array}{c}\phi_{1}^{2}=1 \\
\phi_{2}^{2}=1+\phi_{3}+\phi_{4} \\
\phi_{3}^{2}=1+\phi_{1}+\phi_{4} \\
\phi_{4}^{2}=1+\phi_{1}+\phi_{3} \\
\phi_{5}^{2}=1+\phi_{3}+\phi_{4} \\
\phi_{1} \phi_{2}=\phi_{5}, \phi_{1} \phi_{3}=\phi_{3} \\
\phi_{1} \phi_{4}=\phi_{4}, \phi_{1} \phi_{5}=\phi_{2} \\
\phi_{2} \phi_{3}=\phi_{2}+\phi_{5} \\
\phi_{2} \phi_{4}=\phi_{2}+\phi_{5} \\
\phi_{2} \phi_{5}=\phi_{1}+\phi_{3}+\phi_{4} \\
\phi_{3} \phi_{4}=\phi_{3}+\phi_{4} \\
\phi_{3} \phi_{5}=\phi_{2}+\phi_{5} \\
\phi_{4} \phi_{5}=\phi_{2}+\phi_{5}\end{array}$ & 0 & $S O(5)_{2}$ \\
\hline
\end{tabular}

